\title{
A Multilevel and Single-Stage Trellis Coded Modulation Scheme
}

\author{
Jia-Yin Wang and Mao-Chao Lin \\ Department of Electrical Engineering, National Taiwan University, ROC
}

Abstract : We propose a very powerful trellis coded modulation (TCM) scheme which has the structure of multilevel and single-stage coding. For the proposed TCM scheme, the encoder consists of a binary convolutional encoder which is followed by a multilevel delay processor and a signal mapper. By computer search, we find many useful TCM systems which are good for AWGN channels.

\section{Introduction}

Multilevel coding [1-5] is a frequently used technique in designing coded modulation systems. Suppose that there are $2^{m}$ signal points in a signal constellation. Then, the signal constellation can be partitioned into an $m$-level structure. In the conventional multilevel coding, $m$ binary codes are used for encoding the $m$ levels, where the $m$ binary codes may not necessarily be distinct. We may say that the system has an $m$-level and $m$-stage coding structure.

In section II, we propose a multilevel trellis coded modulation (TCM) scheme for which a single $(m, r)$ binary convolutional code $C$ is used for encoding and decoding. We say that such a system has a multilevel and singlestage coding structure. At each time unit, $r$ message bits are encoded into $m$ code bits. Through a multilevel delay processor, various time delays are introduced to these $m$ code bits. The output of the multilevel delay processor is a binary $m$-tuple which is then mapped into a signal point of the signal constellation through a signal mapper. The decoding for the proposed TCM is described in section III. In section IV, many TCM systems which are good for AWGN channels are found by computer search. Simulation and theoretical analysis are provided for some TCM systems. Finally, conclusions are given in section $\mathrm{V}$.

\section{The Proposed Trellis Coded Modulation Scheme}

The original TCM scheme proposed by Ungerboeck [6] uses the concept of set partitioning of a signal constellation and does not have the multilevel coding structure. In [2], Imai proposed a TCM which uses the concept of set partitioning of a signal constellation and has a multilevel and multistage coding structure. We now propose a new TCM which also uses the concept of set partitioning of a signal constellation and however has a multilevel and single-stage coding structure.

Let $W_{0}$ be a constellation which consists of $2^{m}$ signal points. Each signal point $\tilde{\omega}(\tilde{s})$ in $W_{0}$ can be labelled by $m$ bits, i.e., $\tilde{s}=\left(s_{1}, s_{2}, \ldots, s_{m}\right)$. Suppose that we want to construct a TCM based on $W_{0}$ which has a coding rate of $r$ information bits per signal point, where $1 \leq r \leq m-$ 1. An $(m, r)$ binary convolutional code $C$ will be used. The encoding for the proposed TCM is shown in Fig. 1. At the $t$-th time unit of encoding, an $r$-bit information

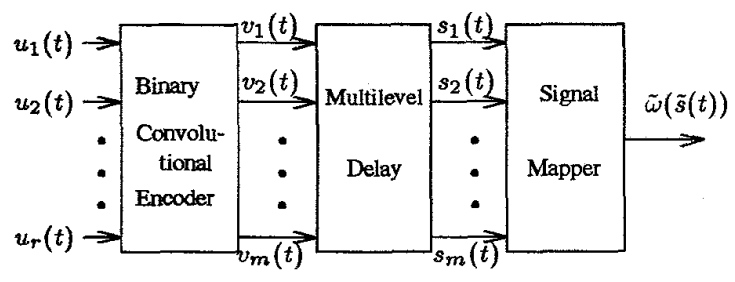

Figure 1: Encoding of the proposed TCM

$\tilde{u}(t)=\left(u_{1}(t), u_{2}(t), \cdots, u_{r}(t)\right)$ is fed into the encoder of $C$ and an $m$-bit code branch $\tilde{v}(t)=\left(v_{1}(t), v_{2}(t), \cdots, v_{m}(t)\right)$ is produced by the encoder of $C$. The output of the encoder of $\mathrm{C}$ is fed into a multilevel delay processor for which the output is an $m$-tuple $\tilde{s}(t)=\left(s_{1}(t), s_{2}(t), \cdots s_{m}(t)\right)$ such that

$$
s_{l}(t)=v_{l}\left(t-\sum_{i=l}^{m} \lambda_{i}\right), \quad l=1, \cdots, m,
$$

where $\lambda_{l}, l=1,2, \cdots, m$ are delay constants and $\lambda_{m}=0$. The $m$-tuple $\tilde{s}(t)$ is then mapped into a signal point $\tilde{\omega}(\tilde{s}(t))$ in $W_{0}$ by a signal mapper.

In the following, we show a specific design of TCM which can take advantage of the introduced multilevel delay processor. Let $x_{1}=\left(s_{1}, s_{2}, \cdots, s_{p_{1}}\right), x_{2}=$ $\left(s_{p_{1}+1}, s_{p_{1}+2}, \cdots, s_{p_{2}}\right), \cdots, x_{q}=\left(s_{p_{q-1}+1}, s_{p_{q-1}+2}, \cdots, s_{p_{q}}\right)$, where $1 \leq q \leq m$ and $p_{q}=m$. In this way, we have $\tilde{s}=\left(s_{1}, s_{2}, \cdots, s_{m}\right)=\left(x_{1}, x_{2}, \cdots, x_{q}\right)$. Let $\tilde{s}^{\prime}=$ $\left(s_{1}^{\prime}, s_{2}^{\prime}, \cdots, s_{m}^{\prime}\right)=\left(x_{1}^{\prime}, x_{2}^{\prime}, \cdots, x_{q}^{\prime}\right)$. Let $D^{2}\left(\tilde{\omega}(\tilde{s}), \tilde{\omega}\left(\tilde{s}^{\prime}\right)\right)$ denote the Squared Euclidean Distance (SED) between two 
signal points $\tilde{\omega}(\tilde{s})$ and $\tilde{\omega}\left(\tilde{s}^{\prime}\right)$. We define

$$
\begin{aligned}
& \delta_{j}^{2}\left(x_{j}=a, x_{j}^{\prime}=b\right)= \\
& \left\{\begin{array}{r}
\min _{x_{j}=a, x_{j}^{\prime}=b}\left\{D^{2}\left(\tilde{\omega}(\tilde{s}), \tilde{\omega}\left(\tilde{s}^{\prime}\right)\right): \tilde{\omega}(\tilde{s}), \tilde{\omega}\left(\tilde{s}^{\prime}\right) \in W_{0}\right\}, j=1 \\
\min _{x_{j}=a, x_{j}^{\prime}=b}\left\{D^{2}\left(\tilde{\omega}(\tilde{s}), \tilde{\omega}\left(\tilde{s}^{\prime}\right)\right): \tilde{\omega}(\tilde{s}), \tilde{\omega}\left(\tilde{s}^{\prime}\right) \in W_{0},\right. \\
\text { and } \left.x_{i}=x_{i}^{\prime} \text { for } 1 \leq i<j-1\right\}, \quad 1<j \leq q .
\end{array}\right.
\end{aligned}
$$

In this way, we partition $W_{0}$ into a $q$-level structure.

Example 1 : Consider the 8PSK constellation as given in Fig. 2. Let $x_{1}=s_{1}, x_{2}=s_{2}$ and $x_{3}=s_{3}$. We have $\delta_{1}^{2}\left(x_{1}=0, x_{1}^{\prime}=0\right)=0, \delta_{1}^{2}\left(x_{1}=0, x_{1}^{\prime}=1\right)=0.586$, $\delta_{2}^{2}\left(x_{2}=0, x_{2}^{\prime}=1\right)=2, \delta_{3}^{2}\left(x_{3}=0, x_{3}^{\prime}=1\right)=4$.

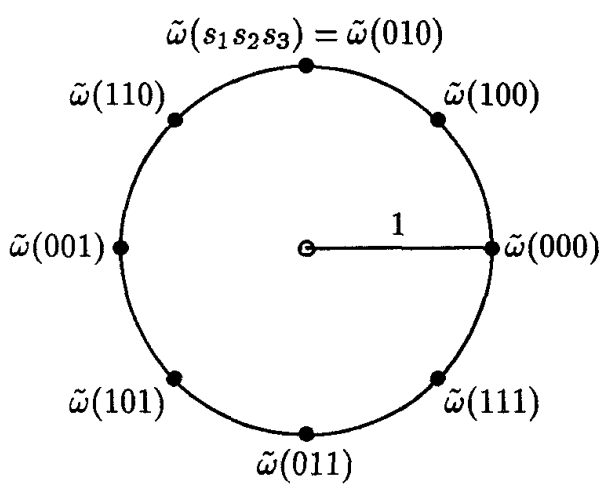

Figure 2: 8PSK signal constellations.

Example 2 ; Let $x_{1}=s_{1}, x_{2}=\left(s_{2}, s_{3}\right)$. We have $\delta_{1}^{2}\left(x_{1}=\right.$ $\left.0, x_{1}^{\prime}=0\right)=0, \delta_{1}^{2}\left(x_{1}=0, x_{1}^{\prime}=1\right)=0.586, \delta_{2}^{2}\left(x_{2}=\right.$ $\left.(00), x_{2}^{\prime}=(10)\right)=2, \delta_{2}^{2}\left(x_{2}=(00), x_{2}^{\prime}=(11)\right)=2$, and $\delta_{2}^{2}\left(x_{2}=(00), x_{2}^{\prime}=(01)\right)=4$.

With the $q$-level $W_{0}$, we can design a $q$-level and singlestage TCM. We set the delay time constants to be

$$
\lambda_{l}= \begin{cases}0, & p_{j-1}+1 \leq l<p_{j} \text { and } 1 \leq j \leq q \\ \lambda, & l=p_{j} \text { and } 1 \leq j \leq q-1\end{cases}
$$

where $p_{0}=0$ and $\lambda$ is a constant. Write $y_{1}=$ $\left(v_{1}, v_{2}, \cdots, v_{p_{1}}\right), y_{2}=\left(v_{p_{1}+1}, v_{p_{1}+2}, \cdots, v_{p_{2}}\right), \cdots, y_{q}=$ $\left(v_{p_{q-1}+1}, v_{p_{q-1}+2}, \cdots, v_{p_{q}}\right)$. This implies that $\tilde{v}=$ $\left(v_{1}, v_{2}, \cdots, v_{m}\right)=\left(y_{1}, y_{2}, \cdots, y_{q}\right)$. By (1) and (3), we have

$$
x_{j}(t)=y_{j}(t-(q-j) \lambda), \quad j=1, \cdots, q .
$$

Let $\bar{s}=\{\cdots, \tilde{s}(t-1), \tilde{s}(t), \tilde{s}(t+1), \cdots\}$ and $\bar{s}^{\prime}=$ $\left\{\cdots, \tilde{s}^{\prime}(t-1), \tilde{s}^{\prime}(t), \tilde{s}^{\prime}(t+1), \cdots\right\}$ be two distinct $s$ sequences and the associated $v$ sequences be $\bar{v}=\{\cdots, \tilde{v}(t-$ 1), $\tilde{v}(t), \tilde{v}(t+1), \cdots\}$ and $\bar{v}^{\prime}=\left\{\cdots, \tilde{v}^{\prime}(t-1), \tilde{v}^{\prime}(t), \tilde{v}^{\prime}(t+\right.$ $1), \cdots\}$ respectively and the associated symbol sequences be $\bar{\omega}=\{\cdots, \tilde{\omega}(\tilde{s}(t-1)), \tilde{\omega}(\tilde{s}(t)), \tilde{\omega}(\tilde{s}(t+1)), \cdots\}$ and $\bar{\omega}^{\prime}=$ $\left\{\cdots, \tilde{\omega}\left(\tilde{s}^{\prime}(t-1)\right), \tilde{\omega}\left(\tilde{s}^{\prime}(t)\right), \tilde{\omega}\left(\tilde{s}^{\prime}(t+1)\right), \cdots\right\}$ respectively. Assume that $\bar{v}$ and $\bar{v}^{\prime}$ are distinct for some code branches which all occur within $\lambda$ consecutive code branches. This assumption assures that whenever $y_{j}(t) \neq y_{j}^{\prime}(t)$, we have $y_{i}(t-k \lambda)=y_{i}^{\prime}(t-k \lambda)$ for $k \geq 1$ and $1 \leq i \leq q$. Then if $j \geq 2$ and $x_{j}(t+(q-j) \lambda)=y_{j}(t) \neq y_{j}^{\prime}(t)=x_{j}^{\prime}(t+(q-j) \lambda)$, we have $x_{i}(t+(q-j) \lambda)=y_{i}(t-(j-i) \lambda)=y_{i}^{\prime}(t-(j-i) \lambda)=$ $x_{i}^{\prime}(t+(q-j) \lambda)$ for $1 \leq i<j$. It follows from equation (2) that

$$
\begin{aligned}
& D^{2}\left(\tilde{s}(t+(q-j) \lambda), \tilde{s}^{\prime}(t+(q-j) \lambda)\right) \\
& \geq \delta_{j}^{2}\left(x_{j}(t+(q-j) \lambda), x_{j}^{\prime}(t+(q-j) \lambda)\right) \\
& =\delta_{j}^{2}\left(y_{j}(t), y_{j}^{\prime}(t)\right) .
\end{aligned}
$$

The SED between $\bar{\omega}$ and $\bar{\omega}^{\prime}$ is then

$$
\begin{aligned}
D^{2}\left(\bar{\omega}, \bar{\omega}^{\prime}\right) & \geq \sum_{t} \sum_{j=1}^{q} \delta_{j}^{2}\left(x_{j}(t+(q-j) \lambda), x_{j}^{\prime}(t+(q-j) \lambda)\right) \\
& =\sum_{t} \sum_{j=1}^{q} \delta_{j}^{2}\left(y_{j}(t), y_{j}^{\prime}(t)\right) .
\end{aligned}
$$

Example 3 : Let $m=3$ and $\tilde{v}=(\cdots,(000),(000),(000)$, $\cdots)$ and $\bar{v}^{\prime}=\left(\cdots,(000), \tilde{v}^{\prime}(t)=(100), \tilde{v}^{\prime}(t+1)=(110)\right.$, $\left.\tilde{v}^{\prime}(t+2)=(111),(000), \cdots\right)$. Let $x_{1}=s_{1}, x_{2}=s_{2}, x_{3}=$ $s_{3}$. Set $\lambda=3$, the sequences $\vec{v}$ and $\bar{v}^{\prime}$ are converted into sequences $\vec{s}$ and $\bar{s}^{\prime}$, which are respectively given by

$$
\begin{aligned}
& \bar{s}=\left[\begin{array}{ccccccc}
\cdots & 0 & 0 & 0 & 0 & 0 & 0 \\
\cdots & 0 & 0 & 0 & 0 & 0 & 0 \\
\cdots & 0 & 0 & 0 & 0 & 0 & 0
\end{array}\right.
\end{aligned}
$$

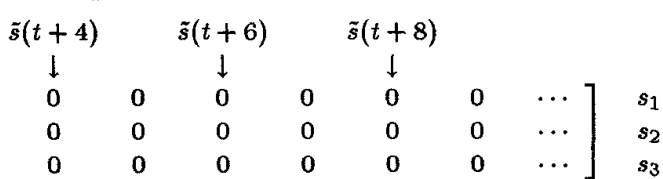

and

$$
\begin{aligned}
& \bar{s}^{\prime}=\left[\begin{array}{ccccccc}
\cdots & & & \tilde{s}^{\prime}(t) & \tilde{s}^{\prime}(t+1) & & \tilde{s}^{\prime}(t+3) \\
\cdots & 0 & 0 & 0 & 1 & & \downarrow \\
\cdots & 0 & 0 & 0 & 0 & 0 & 0 \\
\cdots & 0 & 0 & 0 & 0 & 1 & 0
\end{array}\right.
\end{aligned}
$$

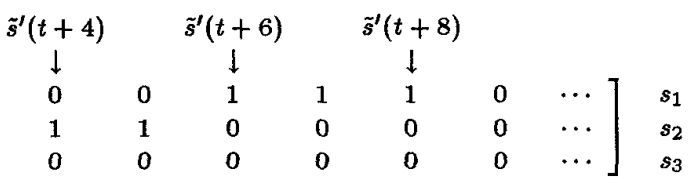

Consider the 8PSK signal constellation. Then, we have $D^{2}\left(\tilde{\omega}(\tilde{s}(t+2)), \tilde{\omega}\left(\tilde{s}^{\prime}(t+2)\right)\right)=\delta_{3}^{2}(1,0)=4, D^{2}(\tilde{\omega}(\tilde{s}(t+$ 4)), $\left.\tilde{\omega}\left(\tilde{s}^{\prime}(t+4)\right)\right)=D^{2}\left(\tilde{\omega}(\tilde{s}(t+5)), \tilde{\omega}\left(\tilde{s}^{\prime}(t+5)\right)\right)=\delta_{2}^{2}(1,0)=$ $2, D^{2}\left(\tilde{\omega}(\tilde{s}(t+i)), \tilde{\omega}\left(\tilde{s}^{\prime}(t+i)\right)\right)=\delta_{1}^{2}(1,0)=0.586$ for $i=$ $6,7,8$. Thus, we have $D^{2}\left(\bar{\omega}, \bar{\omega}^{\prime}\right)=0.586 \times 3+2 \times 2+4 \times 1=$ 9.758 .

Example 4: Suppose that in Example 3, we set $x_{1}=$ $s_{1}, x_{2}=\left(s_{2}, s_{3}\right)$. Then $\bar{v}^{\prime}$ is converted into the sequence $\bar{s}^{\prime}$, where

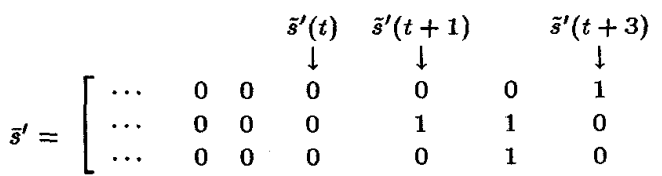




\begin{tabular}{|c|c|c|c|c|c|c|}
\hline$\tilde{s}^{\prime}(t+4)$ & & $\tilde{s}^{\prime}(t+6)$ & & $(t+8)$ & & \\
\hline$\downarrow$ & & $\downarrow$ & & $\downarrow$ & & \\
\hline 1 & 1 & 0 & 0 & 0 & 0 & $\cdots$ \\
\hline 0 & 0 & 0 & 0 & 0 & 0 & $\cdots$ \\
\hline 0 & 0 & 0 & 0 & 0 & 0 & $\cdots$ \\
\hline
\end{tabular}

With this, we have $D^{2}\left(\tilde{\omega}(\tilde{s}(t+1)), \tilde{\omega}\left(\tilde{s}^{\prime}(t+1)\right)\right)=$ $\delta_{2}^{2}((10),(00))=2, \quad D^{2}\left(\tilde{\omega}(\tilde{s}(t+2)), \tilde{\omega}\left(\tilde{s}^{\prime}(t+2)\right)\right)=$ $\delta_{2}^{2}((11),(00))=2, D^{2}\left(\tilde{\omega}(\tilde{s}(t+i)), \tilde{\omega}\left(\tilde{s}^{\prime}(t+i)\right)\right)=\delta_{1}(1,0)$ $=\delta_{1}^{2}(1,0)=0.586$ for $i=3,4,5$. Thus, we have $D^{2}\left(\bar{\omega}, \bar{\omega}^{\prime}\right)$ $=0.586 \times 3+2 \times 2=5.758$.

The squared free distance of this TCM, $D_{\text {free }}^{2}$ is the smallest one of all the possible SED between any pair of two distinct symbol sequences $\bar{\omega}$ and $\bar{\omega}^{\prime}$.

Theorem : If the convolutional code $C$ is not catastrophic, by taking $\lambda$ to be a large enough number, we have

$$
D_{\text {free }}^{2} \geq \min _{\bar{v} \neq \bar{v}^{\prime} ; \bar{v}, \bar{v}^{\prime} \in C}\left\{\sum_{t} \sum_{j=1}^{q} \delta_{j}^{2}\left(y_{j}(t), y_{j}^{\prime}(t)\right)\right\}
$$

proof : We consider the following two conditions.

(i) The sequences $\bar{v}$ and $\bar{v}^{\prime}$ leave a common state in the trellis of $C$ and then rejoin a common state within $\lambda$ consecutive code branches. In this case, all the distinct code branches between $\bar{v}$ and $\vec{v}^{\prime}$ are within $\lambda$ consecutive code branches. Hence, the assumption for deriving equation (6) is met. Thus, $D^{2}\left(\bar{\omega}, \bar{\omega}^{\prime}\right)$ is no less than the righthand side of equation (7).

(ii) The sequences $\bar{v}$ and $\bar{v}^{\prime}$ leave a common state in the trellis of $C$ and did not rejoin a common state within $\lambda$ consecutive code branches. Since $C$ is not catastrophic, the number of distinct code branches, $d(\lambda)$, between $\bar{v}$ and $\bar{v}^{\prime}$ within $\lambda$ consecutive code branches can be increased to a large enough value such that $\min _{y_{1} \neq y_{1}^{\prime}}\left\{\delta_{1}^{2}\left(y_{1}, y_{1}^{\prime}\right)\right\} \cdot d(\lambda)$ will be no less than the righthand side of equation (7) by taking $\lambda$ to be a large enough value. Since the number of distinct symbols between $\bar{\omega}$ and $\bar{\omega}^{\prime}$ is at least $d(\lambda)$, we see that $D^{2}\left(\bar{\omega}, \bar{\omega}^{\prime}\right)$ is at least $\min _{y_{1} \neq y_{1}^{\prime}}\left\{\delta_{1}^{2}\left(y_{1}, y_{1}^{\prime}\right)\right\} \cdot d(\lambda)$.

By combining conditions (i) and (ii), we complete the proof.

$\triangle \triangle$

Note that for $q=1$, the proposed TCM becomes the Ungerboeck's TCM [6] which does not have a multilevel coding structure, and for $q=m$, the proposed TCM becomes the TCM proposed by Hellstern [7].

\section{Decoding}

To decode this TCM, the trellis for the convolutional code $C$ must be used. If the number of encoder memory bits for $C$ is $\nu$, then the number of states for the associated trellis is $2^{\nu}$. Here, we set the truncation length of decoding the binary convolutional code $C$ to be $\lambda$. Let $\bar{z}=\{\cdots, \tilde{z}(t-$
1), $\tilde{z}(t), \tilde{z}(t+1), \cdots\}$ be the received signal sequence, where $\tilde{z}(t)$ is the noise-corrupted form of a transmitted symbol $\tilde{s}(t)$. At the $(t+(q-1) \lambda)$-th time unit of decoding, $\cdots, \tilde{z}(t+$ $(q-1) \lambda-2), \tilde{z}(t+(q-1) \lambda-1)$, and $\tilde{z}(t+(q-1) \lambda)$ are already received. We assume that $\tilde{v}(t-i)=\left(y_{1}(t-i), \cdots\right.$, $\left.y_{m}(t-i)\right)$, has already been correctly recovered for $i \geq \lambda$. The decoding procedure is as follows.

Step 1 : For each of the $2^{p_{1}}$ possible $y_{1}(t)$, we calculate the associated metric $M_{y_{1}(t)}$, which is calculated by

$$
\min _{x_{1}=y_{1}(t)}\left\{D^{2}(\tilde{z}(t+(q-1) \lambda), \tilde{\omega}(\tilde{s}))\right\}
$$

where $\tilde{\boldsymbol{s}}=\left(x_{1}, \cdots, x_{q}\right)$ and $\tilde{\omega}(\tilde{s}) \in W_{0}$.

Step 2 : For each of the $2^{p_{2}}$ possible $y_{2}(t)$, we calculate the associated metric $M_{y_{2}(t)}$. Since $y_{1}(t-\lambda)$ is assumed to have been correctly decoded, we can calculate $M_{y_{2}}(t)$ by

$$
\min _{\substack{x_{2}=y_{2}(t) \\ x_{1}=y_{1}(t-\lambda)}}\left\{D^{2}(\tilde{z}(t+(q-2) \lambda), \tilde{\omega}(\tilde{s}))\right\}
$$

In general, for $1 \leq j \leq q$, we have

Step $\mathbf{j}$ : For each of the $2^{p_{j}}$ possible $y_{j}(t)$, we calculate the associated metric $M_{y_{j}(t)}$, which is calculated by

$$
\min _{\substack{x_{j}=y_{j}(t) \\ x_{1}=y_{1}(t-(j-1) \lambda), \cdots, x_{j-1}=y_{j-1}(t-\lambda)}}\left\{D^{2}(\tilde{z}(t+(q-j) \lambda), \tilde{\omega}(\tilde{s}))\right\}
$$

Step $\mathbf{q}+1$ : By summing one of the $2^{p_{1}}$ possible $M_{y_{1}(t)}$, one of the $2^{p_{2}}$ possible $M_{y_{2}(t)}, \cdots$ and one of $2^{p_{q}}$ possible $M_{y_{q}(t)}$, we can compute one of the $2^{m}$ possible branch metrics $M_{\tilde{v}(t)}$.

Step q+2 : With $\cdots, M_{\tilde{v}(t-1)}, M_{\tilde{v}(t)}$, and the $2^{\nu}$-state trellis for $C$, we can apply the Viterbi algorithm to recover $\tilde{v}(t-\lambda+1)$ and $\tilde{u}(t-\lambda+1)$. The decoding procedure is then back to step 1 .

Note that the first encoded message $\tilde{u}(1)$ is recovered in the $q \lambda$-th time unit of decoding. Compared to the TCM used by Ungerboeck [6] with the same trellis complexity, our TCM requires an additional decoding delay of $(q-1) \lambda$ time units.

\section{Performance Analysis}

Using computer search, we find many good 8PSK TCM which are listed in Table 1. Each TCM in Table 1 has the coding rate of 2 information bits per symbol. We also find many good 16QAM TCM which are listed in Table 2. Each TCM in Table 2 has the coding rate of 3 information bits per symbol. In these tables, $N$ represents the distance spectrum and $N_{I}$ represents the information-weight spectrum, where $N\left(D^{2}\right)$ is the number of neighbors at an SED of $D^{2}$ from a given symbol sequence and $N_{I}\left(D^{2}\right)$ is the total number of information bits contained in all the $N\left(D^{2}\right)$ 
neighbors at an SED of $D^{2}$ from a given symbol sequence.

Table 1: 8PSK TCM

\begin{tabular}{|c|c|c|c|}
\hline \multirow{3}{*}{$\begin{array}{c}\text { Code 1a } \\
q=3 \\
\{1,2,3\} \\
\end{array}$} & $\nu=2$ & $\overline{D^{2}}$ & 6.34 \\
\hline & \multirow{2}{*}{$G=\left(\begin{array}{lll}1 & 1 & 1 \\
5 & 7 & 0\end{array}\right)$} & $N_{I}$ & 128 \\
\hline & & $N$ & 64 \\
\hline \multirow{3}{*}{$\begin{array}{c}\text { Code } 1 \mathrm{~b} \\
q=3 \\
\{1,2,3\}\end{array}$} & $\nu=3$ & $D^{2}$ & 7.52 \\
\hline & \multirow{2}{*}{$G=\left(\begin{array}{lll}1 & 3 & 2 \\
7 & 4 & 1\end{array}\right)$} & $N_{I}$ & 2048 \\
\hline & & $N$ & 256 \\
\hline \multirow{3}{*}{$\begin{array}{c}\text { Code 1c } \\
q=3 \\
\{1,2,3\} \\
\end{array}$} & $\nu=4$ & $D^{2}$ & 8.93 \\
\hline & \multirow{2}{*}{$G=\left(\begin{array}{lll}4 & 7 & 3 \\
5 & 3 & 4\end{array}\right)$} & $\overline{N_{I}}$ & 5120 \\
\hline & & $N$ & 832 \\
\hline \multirow{3}{*}{$\begin{array}{c}\text { Code } 1 \mathrm{~d} \\
q=2 \\
\{1,(2,3)\}\end{array}$} & $\nu=2$ & $D^{2}$ & 5.17 \\
\hline & \multirow{2}{*}{$G=\left(\begin{array}{lll}0 & 1 & 2\end{array}\right)$} & $N_{I}$ & 32 \\
\hline & & $N$ & 12 \\
\hline \multirow{3}{*}{$\begin{array}{c}\text { Code 1e } \\
q=2 \\
\{1,(2,3)\}\end{array}$} & $\nu=3$ & $\bar{D}^{2}$ & 5.76 \\
\hline & \multirow{2}{*}{$G=\left(\begin{array}{lll}2 & 2 & 1 \\
7 & 5 & 4\end{array}\right)$} & $N_{I}$ & 8 \\
\hline & & $N$ & 8 \\
\hline \multirow{3}{*}{$\begin{array}{c}\text { Code 1f } \\
q=2 \\
\{1,(2,3)\}\end{array}$} & $\nu=4$ & $D^{2}$ & 7.17 \\
\hline & \multirow{2}{*}{$G=\left(\begin{array}{lll}5 & 7 & 5 \\
3 & 3 & 4\end{array}\right)$} & $N_{I}$ & 24 \\
\hline & & $N$ & 12 \\
\hline
\end{tabular}

From Table 1, we see that, compared to the uncoded QPSK, the 8-state 8PSK TCM with $q=1,2$ and 3 can achieve asymptotic coding gains of $3.6 \mathrm{~dB}, 4.6 \mathrm{~dB}$, and 5.75 $\mathrm{dB}$ respectively for AWGN channels. Note that $q=1$ is the Ungerboeck's TCM case. From Table 2, we see that, compared to the uncoded 8AMPM, the 4-state 16QAM TCM with $q=1,2,3$ and 4 can achieve asymptotic coding gains of $3.0 \mathrm{~dB}, 4.77 \mathrm{~dB}, 6.0 \mathrm{~dB}$ and $6.0 \mathrm{~dB}$ respectively for AWGN channels. Simulations have been implemented by taking $\lambda=10 \nu$ and the results are given in Fig. 3 and 4 respectively. We see that, compared to the un-

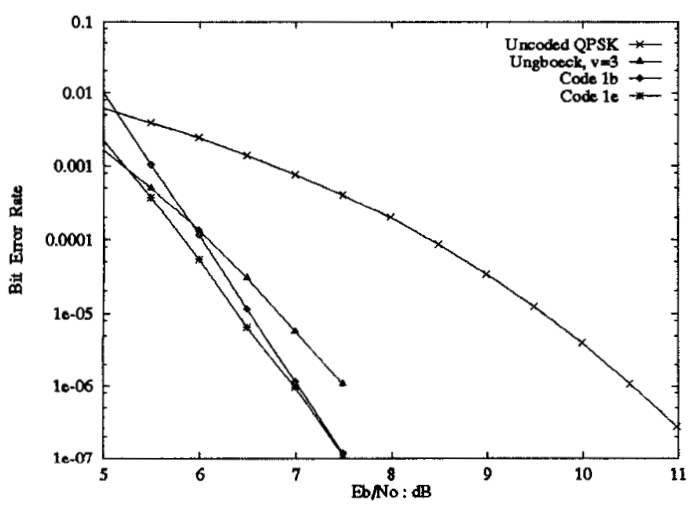

Figure 3: Simulation results for 8PSK TCM.
Table 2: 16QAM TCM

\begin{tabular}{|c|c|c|c|c|c|c|c|}
\hline \multirow{3}{*}{$\begin{array}{c}\text { Code } 2 \mathrm{a} \\
q=4 \\
\{1,2,3,4\}\end{array}$} & \multicolumn{5}{|c|}{$\nu=2$} & \multirow{2}{*}{$\frac{D^{\prime}}{N_{I}}$} & \multirow{2}{*}{$\frac{3.2}{2007}$} \\
\hline & \multirow{2}{*}{$G=$} & & & 1 & 1 & & \\
\hline & & & & $\begin{array}{l}1 \\
2\end{array}$ & 0 & $N$ & 594 \\
\hline Code $2 b$ & \multicolumn{5}{|c|}{$\nu=3$} & $D^{2}$ & 3.6 \\
\hline \multirow{2}{*}{$\begin{array}{c}q=4 \\
\{1,2,3,4\}\end{array}$} & \multirow{2}{*}{$G=$} & \multirow{2}{*}{\multicolumn{2}{|c|}{0}} & \multirow{2}{*}{\multicolumn{2}{|c|}{$\left.\begin{array}{ll}1 & 1 \\
2 & 0 \\
1 & 0\end{array}\right)$}} & $N_{I}$ & 152 \\
\hline & & & & & & $N$ & 152 \\
\hline Code $2 \mathrm{c}$ & \multicolumn{5}{|c|}{$\nu=4$} & $D^{2}$ & 4.0 \\
\hline \multirow{2}{*}{$\begin{array}{c}q=4 \\
\{1,2,3,4\}\end{array}$} & \multirow{2}{*}{$G=$} & \multirow{2}{*}{\multicolumn{2}{|c|}{1}} & \multirow{2}{*}{$\begin{array}{l}1 \\
0 \\
0 \\
\end{array}$} & \multirow{2}{*}{$\left.\begin{array}{l}1 \\
1 \\
4\end{array}\right)$} & $N_{I}$ & 1881 \\
\hline & & & & & & $N$ & 932 \\
\hline Code $2 \mathrm{~d}$ & \multicolumn{5}{|c|}{$\nu=2$} & $D^{2}$ & 3.2 \\
\hline \multirow{2}{*}{$\begin{array}{c}q=\mathbf{3} \\
\{1,2,(3,4)\}\end{array}$} & \multirow{2}{*}{\multicolumn{5}{|c|}{$G=\left(\begin{array}{llll}0 & 0 & 0 & 1 \\
3 & 3 & 1 & 1 \\
3 & 1 & 2 & 2\end{array}\right)$}} & $N_{I}$ & 2049 \\
\hline & & & & & & $N$ & 595 \\
\hline Code 2e & \multicolumn{5}{|c|}{$\nu=3$} & $D^{2}$ & 3.6 \\
\hline \multirow{2}{*}{$\begin{array}{c}q=3 \\
\{1,2,(3,4)\}\end{array}$} & \multirow{2}{*}{$G=$} & \multirow{2}{*}{$\begin{array}{l}2 \\
3 \\
1 \\
\end{array}$} & 3 & \multirow{2}{*}{$\begin{array}{l}1 \\
3 \\
2\end{array}$} & \multirow{2}{*}{$\left.\begin{array}{l}0 \\
1 \\
2\end{array}\right)$} & $\overline{N_{I}}$ & 957 \\
\hline & & & 3 & & & $N$ & 228 \\
\hline \multirow{3}{*}{$\begin{array}{c}\text { Code } 2 f \\
q=3 \\
\{1,2,(3,4)\}\end{array}$} & \multicolumn{5}{|c|}{$\nu=4$} & $D^{2}$ & 4.0 \\
\hline & \multirow{2}{*}{$G=$} & 1 & & \multirow{2}{*}{$\begin{array}{l}3 \\
2 \\
3 \\
\end{array}$} & & $N_{I}$ & 2532 \\
\hline & & 4 & 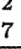 & & & $N$ & 513 \\
\hline Code 2g & \multicolumn{5}{|c|}{$\nu=2$} & $D^{2}$ & 2.4 \\
\hline$q=2$ & & $\begin{array}{l}0 \\
0\end{array}$ & & $\begin{array}{l}0 \\
3\end{array}$ & $\begin{array}{l}1 \\
3\end{array}$ & $N_{I}$ & 110 \\
\hline$\{1,(2,3,4)\}$ & & 3 & 2 & 1 & $\begin{array}{l}3 \\
1 \\
\end{array}$ & $N$ & 34 \\
\hline Code $2 \mathrm{~h}$ & & & $=$ & & & $D^{2}$ & 2.8 \\
\hline$q=2$ & & $\begin{array}{l}0 \\
2\end{array}$ & 0 & 0 & & $N_{I}$ & 67 \\
\hline$\{1,(2,3,4)\}$ & & 7 & 5 & $\begin{array}{l}1 \\
5 \\
\end{array}$ & $\begin{array}{l}1 \\
5 \\
\end{array}$ & $N$ & 35 \\
\hline Code $2 \mathrm{i}$ & & & $=$ & & & $D^{2}$ & 2.8 \\
\hline$q=2$ & & $\begin{array}{l}1 \\
2\end{array}$ & $\begin{array}{l}1 \\
0\end{array}$ & $\begin{array}{l}2 \\
0\end{array}$ & 3 & $N_{I}$ & 14 \\
\hline$\{1,(2,3,4)\}$ & & 6 & 4 & 5 & 1 & $N$ & 6 \\
\hline
\end{tabular}

coded QPSK, the 8-state 8PSK TCM with $q=1,2$ and 3 can achieve coding gains of about $3.0 \mathrm{~dB}, 3.5 \mathrm{~dB}$, and $3.5 \mathrm{~dB}$ respectively at $\mathrm{BER}=10^{-6}$. Moreover, compared to the uncoded 8AMPM, the 4-state 16QAM TCM with $q=1,2,3$ and 4 can achieve coding gains of about $3.0 \mathrm{~dB}$, $3.3 \mathrm{~dB}, 3.8 \mathrm{~dB}$ and $3.8 \mathrm{~dB}$ respectively at $\mathrm{BER}=10^{-6}$. Some theoretical values for BER calculated by using the formula

$$
B E R \approx \frac{1}{r} \cdot \sum_{D} N_{I}\left(D^{2}\right) \cdot Q(D / 2 \sigma)
$$

are given in Table 3 . Theoretical values listed in Table 3 are somewhat lower than the simulated values. This outcome is probably due to the phenomenon of error propagation. In general, we can see that very good error performances for AWGN channels can be achieved by using the proposed TCM. The price that we have to pay is the increased decoding delay and the effort of calculating branch metrics. 


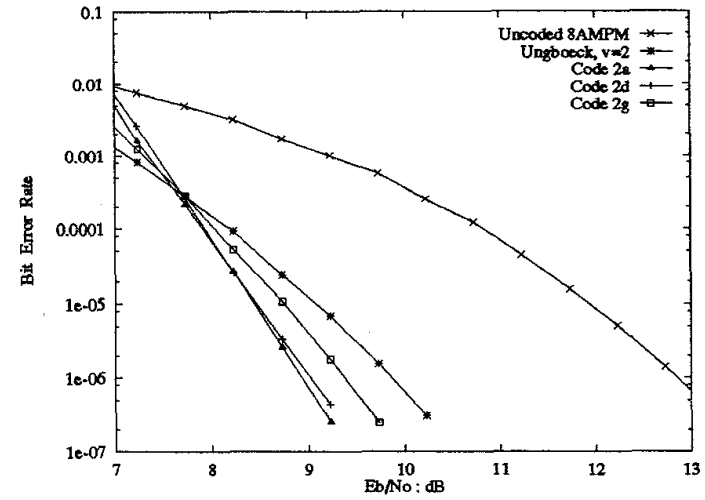

Figure 4: Simulation results for 16QAM TCM.

Table 3: Data obtained from simulation and analysis.

\begin{tabular}{|c|c|c|c|}
\hline & $E_{b} / N_{0}(\mathrm{~dB})$ & Simulation & Analysis \\
\hline Code $1 \mathrm{~b}$ & 6.99 & $1.2 \times 10^{-6}$ & $5.0 \times 10^{-7}$ \\
\hline Code $1 \mathrm{e}$ & 6.49 & $6.7 \times 10^{-6}$ & $4.3 \times 10^{-6}$ \\
\hline Code $2 \mathrm{a}$ & 8.73 & $2.6 \times 10^{-6}$ & $9.6 \times 10^{-7}$ \\
\hline Code $2 \mathrm{~d}$ & 8.73 & $3.4 \times 10^{-6}$ & $9.8 \times 10^{-7}$ \\
\hline Code $2 \mathrm{~g}$ & 9.23 & $2.2 \times 10^{-6}$ & $8.9 \times 10^{-7}$ \\
\hline
\end{tabular}

\section{Conclusions}

The multilevel and single-stage TCM scheme proposed in this paper is obtained by introducing a multilevel delay processor to the TCM designed by Ungerboeck. This is in fact equivalent to introducing new parameters for designing TCM. With various choices of decoding delays and various numbers of trellis states, we can obtain many useful TCM with various error performances.
Although the analysis of the proposed TCM is concentrated on AWGN channels, it is expected that the proposed TCM with large $q$ are good for the Rayleigh fading channels since large $q$ results in large minimum symbol distance.

\section{References}

[1] H. Imai and S. Hirakawa, "A new multilevel coding method using error-correcting codes," IEEE Trans. Inform. Theory, vol. IT-23, no. 3, pp. 371-376, May 1977.

[2] K. Yamaguchi and H. Imai, "Highly reliable multilevel channel coding system using binary convolutional codes," Electronics Letters, 27th Aug. 1987, vol. 23 , no. 13 , pp. 939-941.

[3] V.V. Ginzburg "Multidimensional signals for a continuous channel," Problemy Peredachi Informatsii, vol. 20, no. 1, pp. 28-46, 1984.

[4] S.I. Sayegh, "A class of optimum block codes in signal space," IEEE Trans. Commun., vol. COM-34, no. 10, pp. 1043-1045, Oct. 1986.

[5] E.L. Cusack, "Error control codes for QAM signaling," Electronics Letters, 19th Jan. 1984, vol. 20, no. 2 , pp. $62-63$.

[6] G. Ungerboeck, "Channel coding with multilevel/phase signals," IEEE Trans. Inform. Theory, vol. IT-28, no. 1, pp. 55-67, Jan. 1982.

[7] Günter Hellstern, "Coded Modulation with Feedback Decoding Trellis Codes," IEEE Conference on Commun., pp. 1071-1075, 1993. 